\title{
Postoperative atrial fibrillation in pneumonectomy for primary lung cancer
}

\author{
Hao Wang ${ }^{1 \#}$, Zhexin Wang $^{2 \#}$, Mengmeng Zhou ${ }^{1 \#}$, Jindong Chen $^{1}$, Feng Yao ${ }^{2 *}$, Liang Zhao ${ }^{1}$, Ben He ${ }^{1 *}$ \\ ${ }^{1}$ Department of Cardiology, Shanghai Chest Hospital, Shanghai Jiao Tong University, Shanghai, China; ${ }^{2}$ Department of Thoracic Surgery, Shanghai \\ Chest Hospital, Shanghai Jiao Tong University, Shanghai, China \\ Contributions: (I) Conception and design: L Zhao, F Yao; (II) Administrative support: B He; (III) Provision of study materials or patients: F Yao, Z \\ Wang, H Wang; (IV) Collection and assembly of data: H Wang, Z Wang, M Zhou, J Chen; (V) Data analysis and interpretation: H Wang, M Zhou, \\ Z Wang, L Zhao; (VI) Manuscript writing: All authors; (VII) Final approval of manuscript: All authors. \\ \#These authors contributed equally to this work. \\ *These authors contributed equally to this work as co-correspondence. \\ Correspondence to: Prof. Liang Zhao, MD, PhD. Department of Cardiology, Shanghai Chest Hospital, Shanghai Jiao Tong University, Shanghai, \\ China. Email: zhaoliang80112@126.com; Feng Yao, MD, PhD. Department of Thoracic Surgery, Shanghai Chest Hospital, Shanghai Jiao Tong \\ University, Shanghai, China. Email: yaofeng6796678@126.com; Ben He, MD, PhD. Department of Cardiology, Shanghai Chest Hospital, Shanghai \\ Jiao Tong University, Shanghai, China. Email: heben241@126.com.
}

Background: This study assessed the incidence and risk factors (RFs) of postoperative atrial fibrillation (POAF) and its impact on clinical outcomes in patients undergoing pneumonectomy for lung cancer.

Methods: Between 2013 and 2018, this monocentric retrospective study enrolled 324 consecutive pneumonectomy patients for primary lung cancer from our institution and 350 lobectomy and 349 segmentectomy cases matched by age, sex and body mass index (BMI). RF for POAF and postoperative death in pneumonectomy patients were assessed by logistic regression, and long-term outcomes after a median follow-up of 30 (range, 2-61) months by Cox proportional hazard model. Electrophysiology study (EPS) files of $30 \mathrm{AF}$ patients with lung resection history were reviewed.

Results: POAF developed more often after pneumonectomy than lobectomy and segmentectomy $(23.2 \%$ vs. $6.6 \%$ vs. $1.4 \%$, respectively; $\mathrm{P}<0.001$ ). Among 75 pneumonectomy patients with POAF, POAF was solitary in 55 patients $(73.3 \%)$ and concurrent with other complications in 3 patients (4\%). POAF risk after pneumonectomy was 4 and 22 times that after lobectomy and segmentectomy, respectively, with age $>60$ years and left atrial diameter (LAd) $\geq 35 \mathrm{~mm}$ as independent predictors. POAF, infection and hemorrhage were independent RFs for perioperative death after pneumonectomy; however, POAF was not RF for longterm death. Pulmonary vein (PV) trigger was identified in 60\% (18/30) of AF patients with lung resection history, with stump PVs being more active than non-stump PVs $(38.2 \%$ vs. $10.5 \%, \mathrm{P}<0.001)$.

Conclusions: Post-pneumonectomy AF, with remarkable incidence, risk and independent predictors including age $>60$ years and LAd $\geq 35 \mathrm{~mm}$, was mostly solitary and possibly secondary to stump and nonstump PV triggers. POAF, along with infection and hemorrhage, was a RF for perioperative death.

Keywords: Postoperative atrial fibrillation (POAF); primary lung cancer; pneumonectomy; lobectomy; segmentectomy

Submitted Apr 21, 2020. Accepted for publication Sep 15, 2020.

doi: $10.21037 /$ jtd-20-1717

View this article at: http://dx.doi.org/10.21037/jtd-20-1717

\section{Introduction}

Lung cancer is the most frequent cause of death from cancer worldwide (1), and surgical resection remains the preferred treatment, especially for early-stage lung cancer (2). Postoperative atrial fibrillation (POAF) is the most common arrhythmia after thoracic surgery, especially after lung 
resection, with reported overall incidence ranging from $6.4 \%$ to $19 \%$, increasing morbidity, mortality, hospital stay and cost (3-5). And identified risk factors (RFs) after lung section included advanced age, blood transfusion, pneumonectomy, cardiac dysfunction, diabetes mellitus and so on (6-9). Most studies about POAF focused on lobectomy and segmentectomy, some of which tended to consider POAF as generally benign and temporary (10). Although a higher risk of POAF in pneumonectomy patients was observed, our knowledge about POAF after pneumonectomy remains quite limited, and currently, it was speculated that a large extent of surgical resection may be the cause (9). One important characteristic of pneumonectomy is that pulmonary circulation is forced to flow through the remaining side of pulmonary vessels, consequently raising vascular wall tension and possibly increasing POAF incidence. However, this has been rarely mentioned before. In addition, previous studies on POAF after pneumonectomy were limited by small sample size $(5,11)$. Therefore, with relatively large sample size, this study was conducted to assess incidence and RFs of POAF and its impact on clinical outcomes in patients undergoing pneumonectomy for primary lung cancer. We present the following article in accordance with The Strengthening the Reporting of Observational Studies in Epidemiology (STROBE) reporting checklist (available at http://dx.doi. org/10.21037/jtd-20-1717).

\section{Methods}

The study population of this retrospective observational monocentric study was enrolled from the database of our institution. The study protocol was approved by the Shanghai Chest Hospital Ethics Committee (ID: KS1846) and individual consent for this retrospective analysis was waived. The study was conducted in accordance with the Declaration of Helsinki (as revised in 2013).

\section{Study population}

\section{Settings}

Medical records of patients undergoing lung resection at our center for primary lung cancer between January 2013 and May 2018 were reviewed. Pneumonectomy patients were enrolled if eligibility criteria were met. The selection process has been summarized in a flow diagram (Figure S1). Lobectomy and segmentectomy patients with matched sex, age and body mass index (BMI) were selected randomly.

\section{Clinical variables and outcome data}

The preoperative variables included age, gender, BMI, smoking history, coronary artery disease, diabetes mellitus, hypertension, symptomatic heart failure, chronic obstructive pulmonary disease (COPD), preoperative chemotherapy history and echocardiography parameters. Intraoperative variables included tumor laterality, surgical approach and pericardial incision. Pathological variables included TNM stage and pathological pattern. The primary outcome was the occurrence of POAF, and secondary outcomes were other postoperative complications. Perioperative mortality was defined as death within 30 days after surgery or later during the same hospitalization.

\section{Eligibility criteria}

Firstly, every enrolled patient underwent surgery for primary lung cancer without metastasis. Secondly, in lobectomy and segmentectomy group, only single lobectomy and single segmentectomy cases were enrolled, and other anatomical resections such as bi-lobectomy and bi-segmentectomy were excluded. Thirdly, patients were excluded if atrial fibrillation (AF) history, severe lung disease, or incomplete medical records were present.

\section{Follow-up}

After surgery, all pneumonectomy patients along with 17 lobectomy patients were transferred to the intensive care unit (ICU), and the other patients were transferred to hospital wards. Continuous ECG monitoring with rhythm-analysis function was used in all patients to assess arrhythmia occurrence. The identified AF episodes were reviewed by cardiologists to avoid misinterpretation, and divergence of opinion was solved by discussion. AF burden was defined as the proportion of $\mathrm{AF}$ duration recorded by ECG monitoring during the period from after surgery to discharge (12). Relevant data such as onset, frequency and duration of POAF were recorded automatically and reviewed by cardiologists. Postoperative complications other than POAF were also documented. And according to occurrence timing, POAF were categorized into solitary (without other complications), concurrent with other complications (i.e., occurring within $24 \mathrm{~h}$ ) and nonconcurrent with other complications (i.e., either $>24 \mathrm{~h}$ before or $>24 \mathrm{~h}$ after other complications). 


\section{Electrophysiology study (EPS)}

Institutional EPS files of AF patients with lung resection history due to primary lung cancer were reviewed. Briefly, a decapolar diagnostic catheter was placed in the coronary sinus and a circular mapping catheter was placed in the left atrium (LA) via transseptal puncture. The LA electroanatomic shell was then constructed and each pulmonary vein (PV) was carefully mapped successively, including remnant PV stumps. Electrograms at or within each PV were documented with a mapping catheter and compared with intracardiac electrograms obtained from the coronary sinus catheter to identify PV triggers. PV trigger was defined as an atrial ectopic focus localized in stump or non-stump PV according to the earliest atrial activity relative to the reference electrogram or the onset of the ectopic $\mathrm{P}$ wave. The role of ectopic beats in initiation of AF was confirmed by onsite recording (13). Circumferential PV ablation was then performed, and $\mathrm{AF}$ was considered originating from $\mathrm{PV}$ if $\mathrm{AF}$ converted to sinus rhythm or other types of atrial tachycardia upon achieving isolation of remaining PVs or stumps. EPS was then repeated to confirm PV isolation.

\section{Statistical analysis}

Data are expressed as mean \pm standard deviation for normally distributed continuous variables, median (range) for abnormally distributed continuous variables, and percentage for discrete variables. Comparisons between variables were conducted by $\chi^{2}$ test for categorical variables; independent-sample $t$-test or one-way ANOVA plus LSD $t$-test for continuous variables with normal distribution; and Mann-Whitney $U$ test or Kruskal-Wallis $H$ test for continuous variables with non-normal distribution. Logistic regression was performed to assess the relationship between baseline or perioperative characteristics and POAF occurrence. In order to exclude confounding effect of nonsignificant variables, multivariate regression only included significant univariate RFs. Event-free survival was estimated by the Kaplan-Meier method and compared by the log-rank test. Cox proportional hazard model was used to determine the relationship between patients' characteristics and longterm outcomes. A P value $<0.05$ was considered statistically significant. Statistical analysis was performed using SPSS version 22.0 (IBM Corp., Armonk, NY, USA) and Stata 14.0 (Stata Corp., TX, USA).

\section{Results}

\section{Baseline and perioperative characteristics}

Baseline characteristics (Table 1) were generally similar among the three groups. A total of 1,023 patients, including 324 pneumonectomy patients, 350 lobectomy patients and 349 segmentectomy patients were enrolled. Most pneumonectomies were performed via open thoracotomy and were left-sided. All other procedures were performed via VATS except for one lobectomy; $31.1 \%$ lobectomies and $58.5 \%$ segmentectomies were leftsided. TNM $\geq$ II a were more prevalent in pneumonectomy group than lobectomy group and segmentectomy group (99.4\% vs. $36.9 \%$ vs. $3.4 \%$, respectively; $\mathrm{P}<0.001$ ). And squamous carcinoma was the major pathological type in pneumonectomy group, while adenocarcinoma predominated in other groups. Notably, intraoperative AF occurred in $8.33 \%(27 / 324)$ pneumonectomy patients after the pulmonary artery was clamped. Pneumonectomy patients had longer postoperative stay.

\section{POAF and non-POAF complications}

POAF incidence was $23.2 \%$ (75/324) for pneumonectomy, $6.6 \%(23 / 350)$ for lobectomy and $1.4 \%(5 / 349)$ for segmentectomy. As shown in Table 2, most patients with POAF restored sinus rhythm pharmacologically, including 56 pneumonectomy patients and 22 non-pneumonectomy patients. Because pneumonectomy patients had remarkably longer hospitalization and most POAF episodes occurred within the first 7 postoperative days (pneumonectomy, 94.7\%, 71/75; non-pneumonectomy, 100\%, 28/28), we focused on AF burden within this period. And pneumonectomy group had higher AF burden than nonpneumonectomy group (median, $3.0 \%$ vs. $1.8 \%$; $\mathrm{P}=0.011$ ). In pneumonectomy group, patients with POAF had longer postoperative (median, 13 vs. 10 days; $\mathrm{P}<0.001$ ) and ICU (median, 4 vs. 2 days; $\mathrm{P}<0.001$ ) stays than those without POAF.

Briefly, non-POAF complications were more varied and occurred more frequently after pneumonectomy (Table 3). Yet POAF was concurrent with other complications in only $4 \%(3 / 75)$ patients after pneumonectomy and $7.1 \%(2 / 28)$ patients after non-pneumonectomy. In univariate logistic regression, POAF developed independently from other complications in pneumonectomy; however, POAF might 
Table 1 Baseline and perioperative characteristics of the patients studied

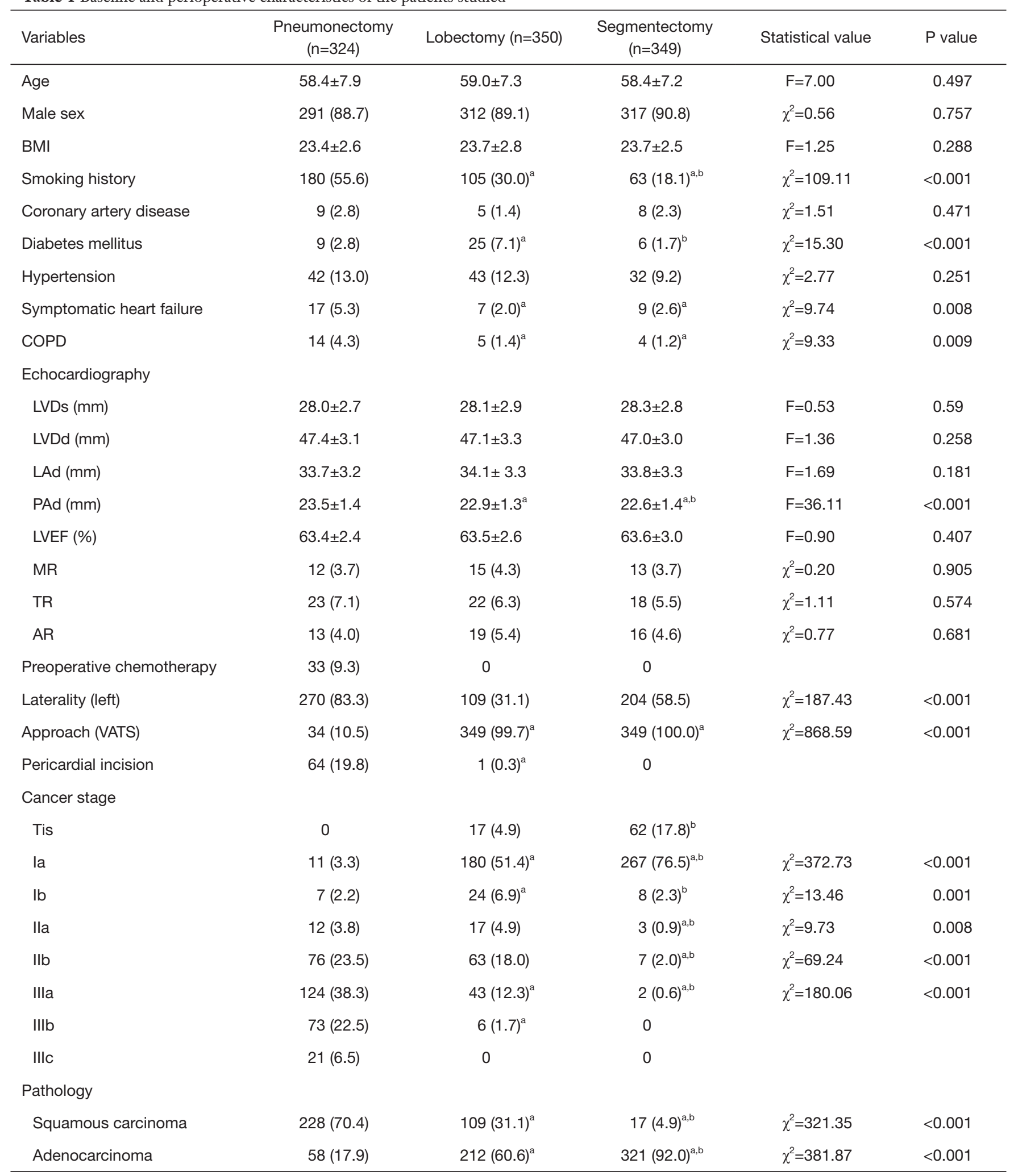

Table 1 (continued) 
Table 1 (continued)

\begin{tabular}{|c|c|c|c|c|c|}
\hline Variables & $\begin{array}{l}\text { Pneumonectomy } \\
\qquad(\mathrm{n}=324)\end{array}$ & Lobectomy $(n=350)$ & $\begin{array}{l}\text { Segmentectomy } \\
\qquad(n=349)\end{array}$ & Statistical value & $P$ value \\
\hline LCLC & $9(2.8)$ & $6(1.7)$ & $2(0.6)^{a}$ & $\chi^{2}=5.01$ & 0.082 \\
\hline Other & $14(4.3)$ & $17(4.9)$ & $8(2.3)$ & $\chi^{2}=3.47$ & 0.176 \\
\hline Blood transfusion & $61(18.8)$ & $14(4)^{a}$ & $5(1.4)^{a, b}$ & $\chi^{2}=81.29$ & $<0.001$ \\
\hline Postoperative stay & $10[3-79]$ & $6[2-145]^{a}$ & $5[2-101]^{a}$ & $\mathrm{H}=497.42$ & $<0.001$ \\
\hline
\end{tabular}

Values are expressed as mean \pm standard deviation, $\mathrm{n}(\%)$ or median [range]. ${ }^{\mathrm{a}}$, vs. pneumonectomy, $\mathrm{P}<0.05 ;{ }^{\mathrm{b}}$, vs. lobectomy, $\mathrm{P}<0.05$. BMI, body mass index; COPD, chronic obstructive pulmonary disease; LVDs, left ventricular end-systolic diameter; LVDd, left ventricular end-diastolic diameter; LAd, left atrial diameter; PAd, pulmonary artery diameter; LVEF, left ventricular ejection fraction; MR, mitral regurgitation; TR, tricuspid regurgitation; AR, aortic regurgitation; VATS, video-assisted thoracoscopic surgery; LCLC, large cell lung cancer; SCLC, small cell lung cancer; POAF, postoperative atrial fibrillation.

possibly be associated with fistula in lobectomy patients $(\mathrm{P}<0.001)$.

Most (10/11) perioperative mortality occurred after pneumonectomy, and notably, POAF occurred in 9 of these 10 pneumonectomy mortalities. After pneumonectomy, AF burden (median, $7.7 \%$ vs. 2.4\%; $\mathrm{P}=0.006$ ) and total $\mathrm{AF}$ duration (median, 13.0 vs. $4.0 \mathrm{~h}$; $\mathrm{P}=0.035$ ) were higher in perioperative death cases than other patients. In pneumonectomy group, all patients with intraoperative AF had POAF occurrence, yet no significant difference was observed in perioperative mortality between patients with and without intraoperative $\mathrm{AF}(17.4 \%$ vs. $9.6 \% ; \mathrm{P}=0.443)$.

\section{Multivariate analysis}

Receiver operating characteristic (ROC) curve was employed to identify optimal cut-off value for continuous variables, and cut-off value for age, left atrial diameter (LAd), left ventricular end-systolic diameter (LVDs) and BMI were $60 \mathrm{y}, 35 \mathrm{~mm}, 29 \mathrm{~mm}$ and $23.6 \mathrm{~kg} / \mathrm{m}^{2}$, respectively. In univariate analysis, POAF risk after pneumonectomy was almost 4 times that after lobectomy [hazard ratio (HR): 4.3; 95\% confidence interval $(\mathrm{CI}): 2.6-7.0 ; \mathrm{P}<0.001]$ and 20 times that after segmentectomy (Figure 1); moreover, POAF risk after lobectomy was 4.8 times that after segmentectomy. Other univariate RFs for POAF included pericardial incision, male sex, age $>60 \mathrm{y}$, symptomatic heart failure, diabetes mellitus, postoperative transfusion, open thoracotomy, left-sided resection, smoking history,
LAd $\geq 35 \mathrm{~mm}$, squamous carcinoma, fistula, infection and hemorrhage. In multivariate analysis (Figure 2), independent RFs for POAF included pneumonectomy, lobectomy, age $>60$ years, diabetes mellitus, LAd $\geq 35 \mathrm{~mm}$, fistula and hemorrhage.

In pneumonectomy patients, univariate $\mathrm{RF}$ for POAF (Figure 1) included age $>60$ years, LAd $\geq 35 \mathrm{~mm}$ and LVDs $>29 \mathrm{~mm}$. In multivariate analysis (Figure 2), independent RFs for POAF included age $>60$ years and LAd $\geq 35 \mathrm{~mm}$. In univariate analysis, RFs for POAF after lobectomy included symptomatic heart failure and fistula (Figure 1); fistula was also an independent RF.

Ten perioperative deaths occurred among the 324 pneumonectomy patients (3.1\%), 9 of which had POAF occurrence. And univariate RFs included age $>60 \mathrm{y}$, symptomatic heart failure, postoperative transfusion, $\mathrm{POAF}$, fistula, infection and hemorrhage. In multivariate analysis, POAF (HR: 16.9; 95\% CI: 1.8-159.7; $\mathrm{P}=0.014)$, infection (HR, 8.8; 95\% CI: 1.4-55.9; $\mathrm{P}=0.021$ ) and hemorrhage (HR: 16.7; 95\% CI: 1.8-159.1; $\mathrm{P}=0.014$ ) were independent $\mathrm{RFs}$ for perioperative death.

\section{Electrophysiological study}

EPS files of 30 patients with AF (19 paroxysmal and 11 persistent) who underwent radiofrequency catheter ablation at our institution between January 2012 and December 2018 were selected. Among the 19 patients with paroxysmal AF, 17 had lobectomy history, and 2 had left pneumonectomy history; while among the 11 patients with persistent $\mathrm{AF}$, 
Table 2 POAF characteristics

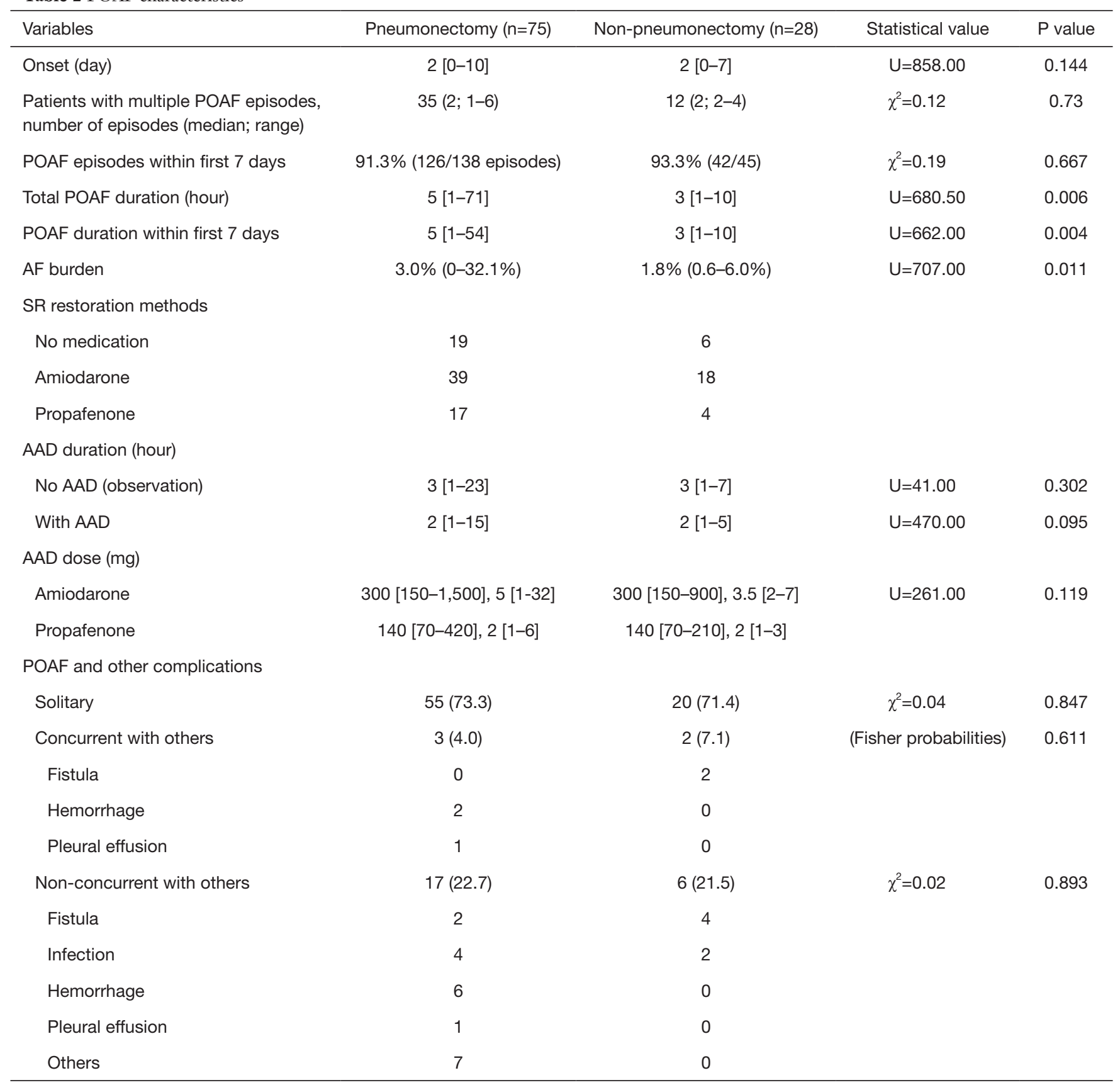

Data are expressed as $\mathrm{n}(\%)$ or median [range]. POAF, postoperative atrial fibrillation; AF, atrial fibrillation; SR, sinus rhythm; AAD, antiarrhythmic drug.

9 had lobectomy and 2 had left pneumonectomy. Lung resection and AF trigger details are summarized in Table 4. Overall, among a total of $120 \mathrm{PVs}$, there were 34 stumps and 86 remaining PVs. PV trigger was identified in 14 stumps of 12 patients and 9 non-stump PVs of 6 patients (examples in Figure 3); and non-PV trigger was identified in the other 12. PV trigger was more active in stump PVs than in non-stump PVs $(13 / 34$ vs. 9/86, $\mathrm{P}<0.001)$. 
Table 3 Non-POAF complication characteristics

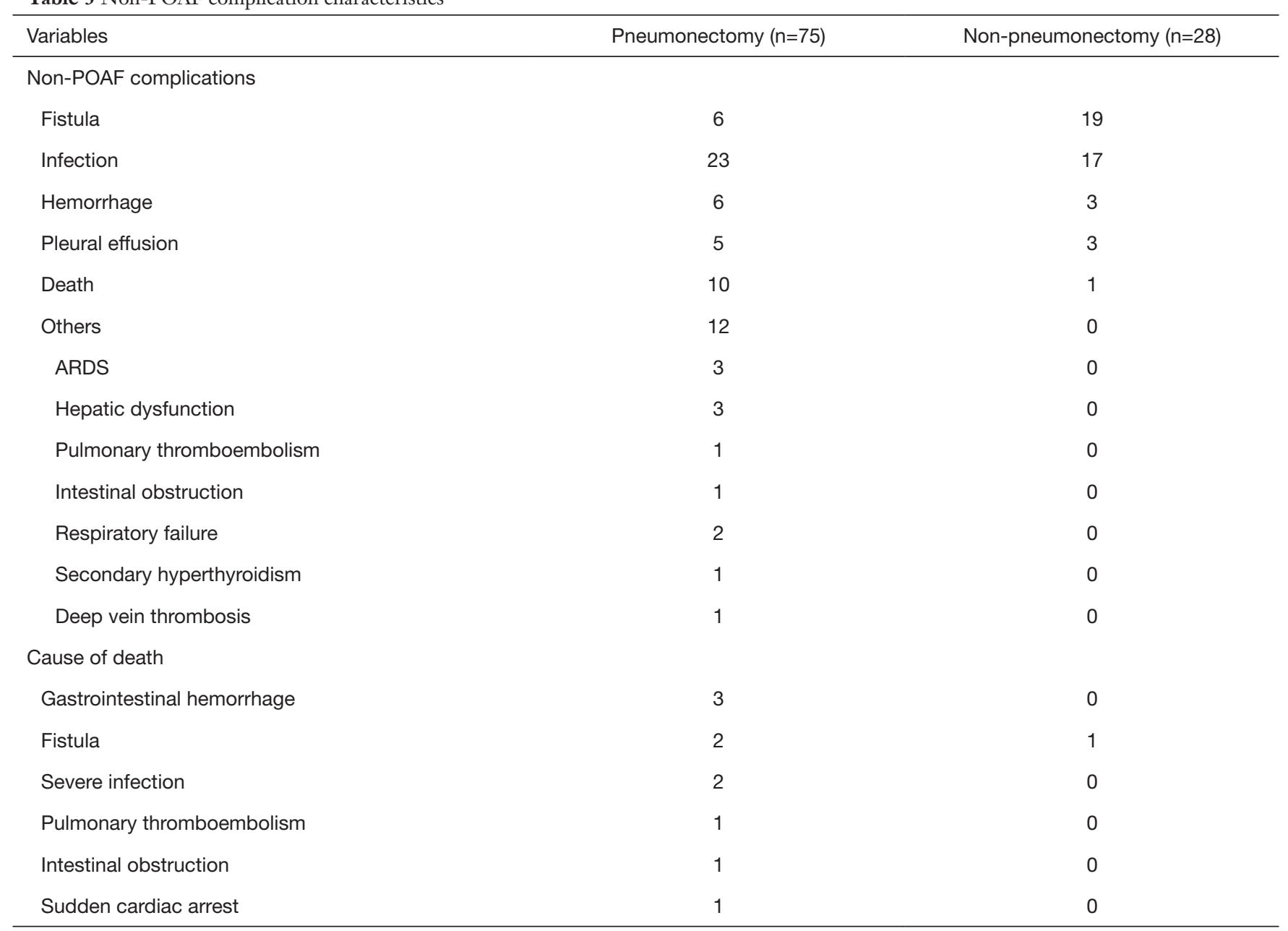

POAF, postoperative atrial fibrillation; ARDS, acute respiratory distress syndrome.

\section{Long-term outcomes}

At median 30 (range, 2-61) months follow-up, 143 of 1,012 patients died after discharge, including 116 pneumonectomy patients, 24 lobectomy patients and 3 segmentectomy patients, with causes including tumor metastasis $(n=113)$, respiratory failure $(n=15)$, stroke $(n=13)$, and intracranial hemorrhage $(n=2)$. The cumulative survival rate was $79.1 \%$, $95.6 \%$, and $98.2 \%$ for pneumonectomy, lobectomy and segmentectomy groups, respectively $(\mathrm{P}<0.001$, Figure 4$)$. In pneumonectomy patients, univariate RFs for postdischarge death included age $>60$ years (HR: 2.0; 95\% CI: 2.0-2.9; $\mathrm{P}<0.001$ ); TNM $\geq$ IIIa (HR: 1.6; 95\% CI: 1.1-2.4; $\mathrm{P}=0.025)$; smoking history (HR: 1.4; $95 \% \mathrm{CI}: 1.0-2.0$; $\mathrm{P}=0.096)$; squamous carcinoma (HR: 1.8 ; 95\% CI: 1.1-2.8; $\mathrm{P}=0.013$ ); and POAF (HR: 1.5; 95\% CI: 1.0-2.2; $\mathrm{P}=0.058$ ); in multivariate analysis, independent RFs were age $>60$ years (HR: 1.9; 95\% CI: $1.3-2.7 ; \mathrm{P}=0.001)$ and $\mathrm{TNM} \geq$ IIIa (HR: 1.8; 95\% CI: 1.2-2.8; P=0.003).

\section{Discussion}

Little is known about POAF after lung resection, while most studies have focused on POAF after cardiac surgery. The present study on POAF after lung resection yielded the following major findings: (I) POAF occurred in $23.2 \%$ of pneumonectomy patients, more often than in lobectomy (6.6\%) and segmentectomy (1.4\%) patients, and mostly as the sole complication; (II) POAF risk after pneumonectomy was almost 4 times that after lobectomy and 22 times that after segmentectomy, with pneumonectomy patients aged $>60$ years or LAd $\geq 35 \mathrm{~mm}$ 
A

\begin{tabular}{|c|c|}
\hline Variables & Risk Ratio $(95 \% \mathrm{Cl})$ \\
\hline Male & $4.07(1.27,13.06)$ \\
\hline Age $>60 y$ & $2.25(1.48,3.42)$ \\
\hline Symptomatic HF & $3.19(1.51,6.73)$ \\
\hline$C A D$ & $2.20(0.67,6.10)$ \\
\hline HBP & $1.57(0.90,2.74)$ \\
\hline DM & $2.18(0.81,5.92)$ \\
\hline Smoking history & $2.06(1.37,3.10)$ \\
\hline COPD & $2.56(0.93,7.04)$ \\
\hline$L A D \geq 35 \mathrm{~mm}$ & $2.52(1.66,3.83)$ \\
\hline LVDs $>29 \mathrm{~mm}$ & $1.58(1.04,2.40)$ \\
\hline $\mathrm{TNM} \geq \| \mathrm{la}$ & $6.78(4.05,11.36)$ \\
\hline Squamous carcinoma & $3.25(2.14,4.94)$ \\
\hline $\mathrm{BM} I \geq 23.6$ & $1.02(0.68,1.53)$ \\
\hline Pericardial incision & $5.22(2.96,9.23)$ \\
\hline Blood transfusion & $2.93(1.66,5.18)$ \\
\hline Open thoracotomy & $5.51(3.59,8.47)$ \\
\hline Left resection & $1.68(1.09,2.60)$ \\
\hline Fistula & $6.68(2.78,16.04)$ \\
\hline Infection & $2.26(1.02,5.04)$ \\
\hline Hemorrhage & $18.91(4.66,76.80)$ \\
\hline $\begin{array}{l}\text { Pleural effusion } \\
\text { Pneumonectomy }\end{array}$ & $\begin{array}{l}1.79(0.21,15.51) \\
20.72(8.26,51.99)\end{array}$ \\
\hline Lobectomy & $4.84(1.82,12.88)$ \\
\hline
\end{tabular}

Variables
B

\begin{tabular}{|c|c|}
\hline Variables & Risk Ratio $(95 \% \mathrm{Cl})$ \\
\hline Male & $5.20(1.21,22.22)$ \\
\hline Age $>60 y$ & $2.65(1.56,4.50)$ \\
\hline Symptomatic HF & $2.46(0.90,6.71)$ \\
\hline CAD & $1.69(0.41,6.91)$ \\
\hline $\mathrm{HBP}$ & $1.39(0.67,2.87)$ \\
\hline DM & $2.75(0.72,10.51)$ \\
\hline Smoking history & $1.10(0.65,1.85)$ \\
\hline COPD & $2.26(0.88,7.81)$ \\
\hline$L A D \geq 35 \mathrm{~mm}$ & $3.22(1.87,5.52)$ \\
\hline LVDs $>29 \mathrm{~mm}$ & $1.97(1.14,3.39)$ \\
\hline $\mathrm{TNM} \geq \| 1 \mathrm{a}$ & $1.06(0.34,3.32)$ \\
\hline Squamous carcinoma & $1.21(0.68,2.15)$ \\
\hline $\mathrm{BM} I \geq 23.6$ & $1.32(0.79,2.23)$ \\
\hline Pericardial incision & $1.58(0.86,2.92)$ \\
\hline Blood transfusion & $1.37(0.73,2.57)$ \\
\hline Open thoracotomy & $1.22(0.54,2.75)$ \\
\hline Left resection & $1.35(0.70,2.61)$ \\
\hline \multicolumn{2}{|l|}{ Fistula } \\
\hline Infection & $1.12(0.43,2.92)$ \\
\hline \multicolumn{2}{|l|}{ Hemorrhage } \\
\hline \multicolumn{2}{|l|}{ Pleural effusion } \\
\hline Lobectomy & \\
\hline
\end{tabular}

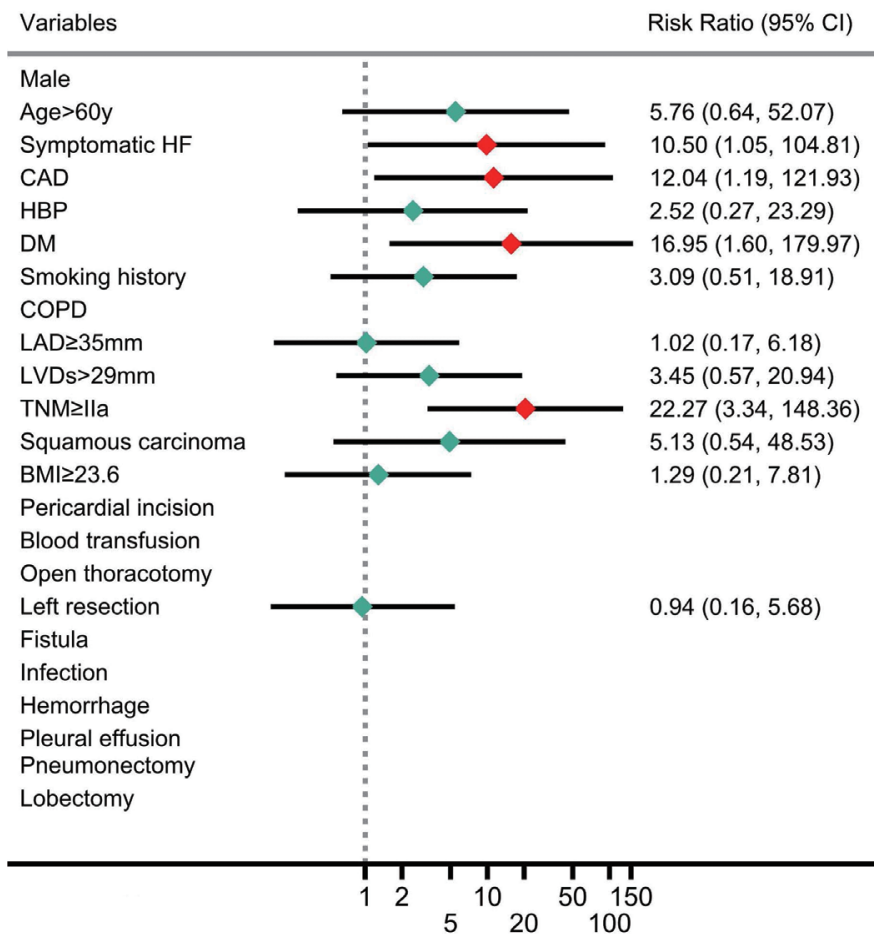

Figure 1 Risk ratio of POAF after lung resection according to variables in univariate logistic regression model. A $\mathrm{P}<0.05$ was considered significant, which was indicated by red diamonds, otherwise turquoise diamonds. (A) All patients, (B) pneumonectomy group, (C) lobectomy group, (D) segmentectomy group. POAF, postoperative atrial fibrillation; CI, confidence interval; HF, heart failure; CAD, coronary artery disease; HBP, high blood pressure; DM, diabetes mellitus; COPD, chronic obstructive pulmonary disease; LAd, left atrial diameter; LVD, left ventricular diameter; BMI, body mass index. 


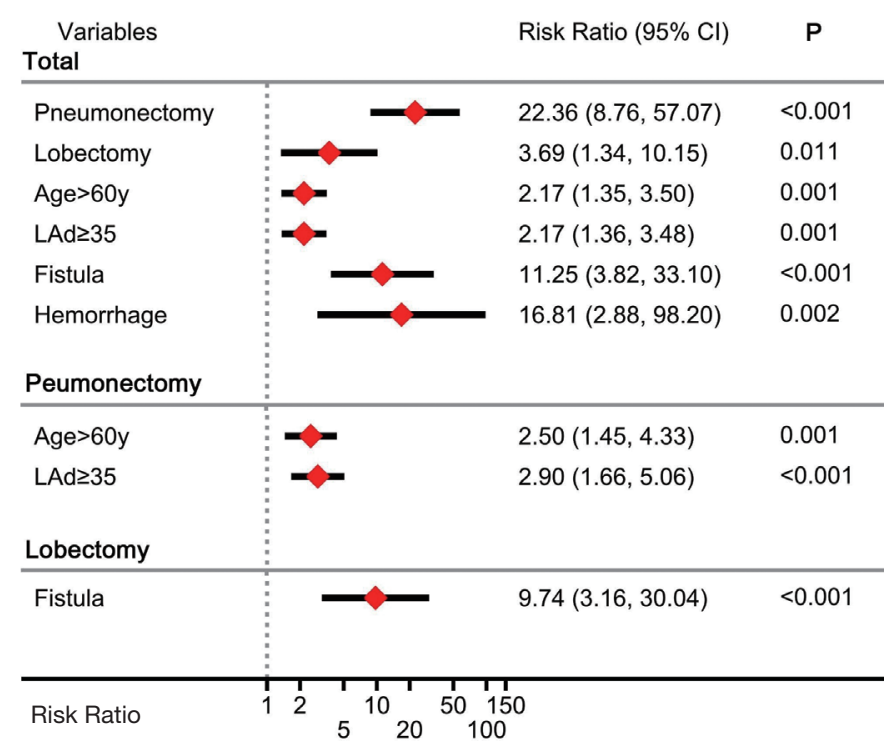

Figure 2 Risk ratio of POAF after lung resection in patients overall and pneumonectomy group according to variables in multivariate logistic regression model. A $\mathrm{P}<0.05$ was considered significant, which was indicated by red diamonds, otherwise turquoise diamonds. POAF, postoperative atrial fibrillation; CI, confidence interval; LAd, left atrial diameter.

Table 4 Trigger and PV characteristics of AF patients with history of lung resection

\begin{tabular}{|c|c|c|c|c|c|c|c|c|c|}
\hline Lung resection & $\mathrm{N}$ & \multicolumn{3}{|c|}{ Patients with AF mechanisms } & \multicolumn{2}{|c|}{ Active PVs } & \multicolumn{3}{|c|}{ Total PVs } \\
\hline \multicolumn{10}{|l|}{$\operatorname{PAF}(n=19)$} \\
\hline Left upper lobectomy & 3 & 2 & 1 (right upper PV) & 0 & 2 & 1 & 3 & 9 & 12 \\
\hline Left lower lobectomy & 6 & 3 & $\begin{array}{c}1 \text { (right upper + } \\
\text { lower PVs) }\end{array}$ & 2 (SVC) & 3 & 2 & 6 & 18 & 24 \\
\hline Right lower lobectomy & 5 & 4 & 1 (left lower PV) & 0 & 4 & 2 & 5 & 15 & 20 \\
\hline Left pneumonectomy & 2 & 1 & $\begin{array}{c}1 \text { (right upper + } \\
\text { lower PVs) }\end{array}$ & 0 & 2 & 2 & 4 & 4 & 8 \\
\hline Summary & 19 & 11 & 5 & 3 & 12 & 8 & 21 & 55 & 76 \\
\hline Left lower lobectomy & 2 & 1 & 0 & $1 \mathrm{TC}$ & 1 & 0 & 2 & 6 & 8 \\
\hline Right upper lobectomy & 1 & 0 & 0 & 1 LAA & 0 & 0 & 1 & 3 & 4 \\
\hline Right lower lobectomy & 4 & 0 & 1 (left-upper PV) & 3 (2 SVC, 1 LAR) & 0 & 1 & 4 & 12 & 16 \\
\hline Left pneumonectomy & 2 & 0 & 0 & 2 unidentified & 0 & 0 & 4 & 4 & 8 \\
\hline Summary & 11 & 1 & 1 & 9 & 1 & 1 & 13 & 31 & 44 \\
\hline Total & 30 & 12 & 6 & 12 & 14 & 9 & 34 & 86 & 120 \\
\hline
\end{tabular}

PV, pulmonary vein; AF, atrial fibrillation; PAF, paroxysmal atrial fibrillation; PerAF, persistent atrial fibrillation; SVC, superior vena cava; CS, coronary sinus; TC, terminal crest; LAA, left atrial appendage; LAR, left atrial roof. 


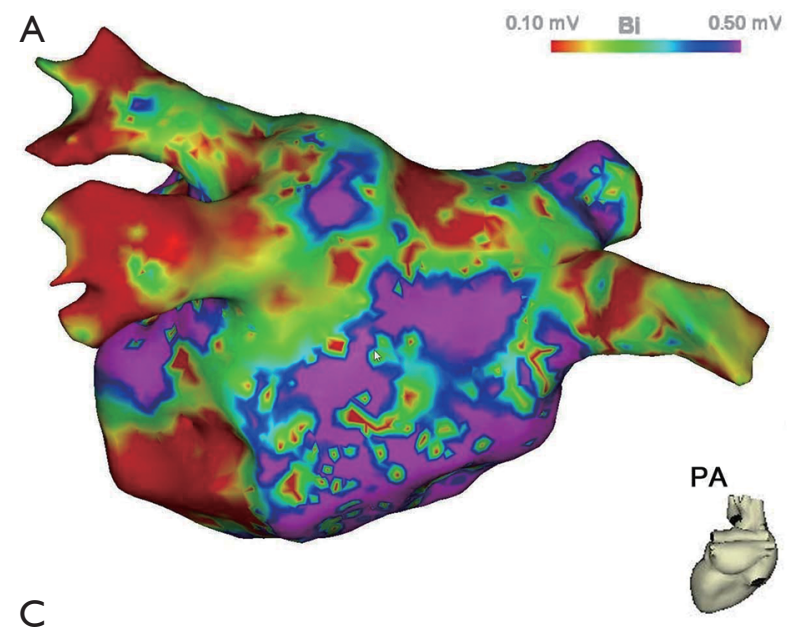

B
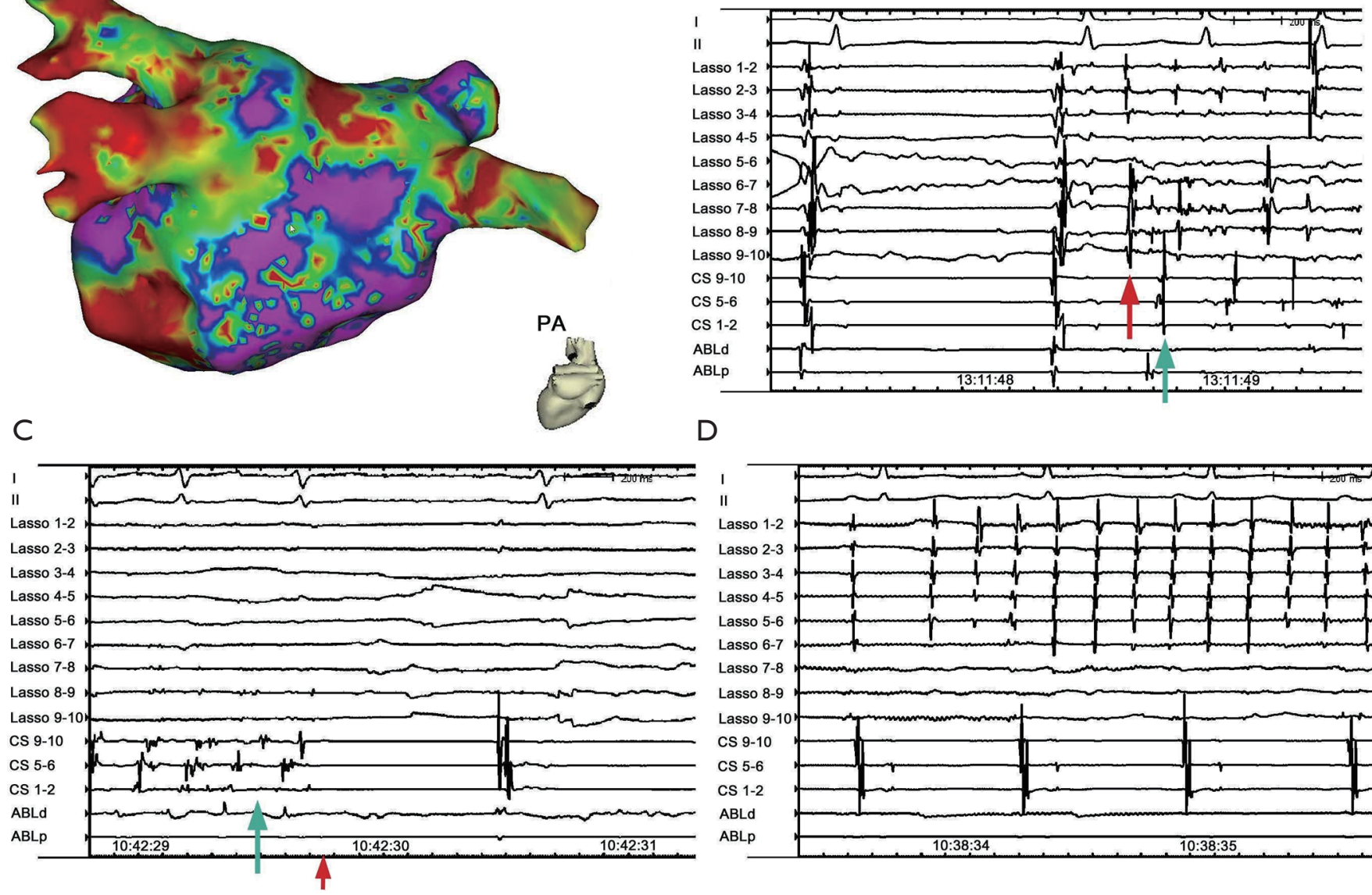

D

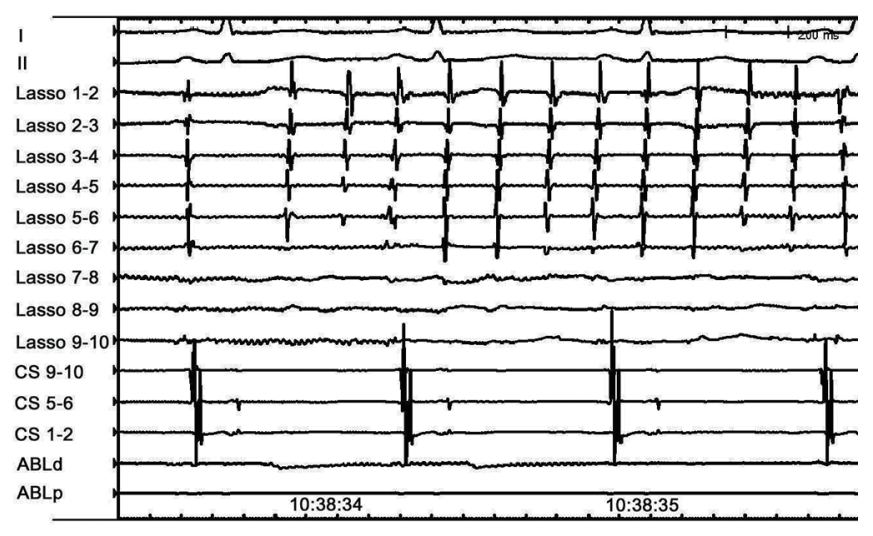

Figure 3 Examples of electrophysiological study files of AF patients with lung resection history. (A) Three-dimensional electroanatomic map of a patient with right-superior lobectomy history in CARTO system of left atria in the posterior-anterior view; (B) AF onset set off by PV trigger from stump PV (right-inferior PV), recorded by a circular mapping catheter (Lasso) in the stump, which was indicated by PV potential (red arrow) preceding atrial potential (turquoise arrow) at AF onset; (C) sinus rhythm was restored right after left PV (non-stump PV) isolation in a patient with left-superior lobectomy history. Turquoise arrow indicated atrial fibrillation recorded by CS catheter; red arrow indicated PV isolation completion; (D) after right PV isolation (non-stump PV), active PV potential recorded by a circular mapping catheter placed within right-superior PV and simultaneous sinus rhythm recorded by CS catheter, indicating that $\mathrm{AF}$ originated from rightsuperior PV. Lasso indicates PV potential recorded by a circular mapping catheter. AF, atrial fibrillation; PV, pulmonary vein; CS, coronary sinus; ABLd, distal ablation catheter potential; ABLp, proximal ablation catheter potential.

being at higher risk for POAF; (III) POAF, along with infection and hemorrhage, may be independent predictors of perioperative death after pneumonectomy; and, (IV) active PV potentials originating from stump and nonstump PVs might be a potential mechanism for POAF in pneumonectomy.

\section{Independence of $\mathrm{POAF}$}

Regardless of the surgical target organ, one can posit that the occurrence of POAF results from superimposition of acute surgery-related factors on pre-existing atrial substrate which is vulnerable to AF initiation and maintenance (14), and for POAF to occur independently of other postoperative complications. In our study, most POAF in pneumonectomy occurred as the sole postoperative complication, which is consistent with a previous study (3) indicating that mechanism underlying POAF in pneumonectomy possibly involved more than inflammation and autonomous nerve system activation. 


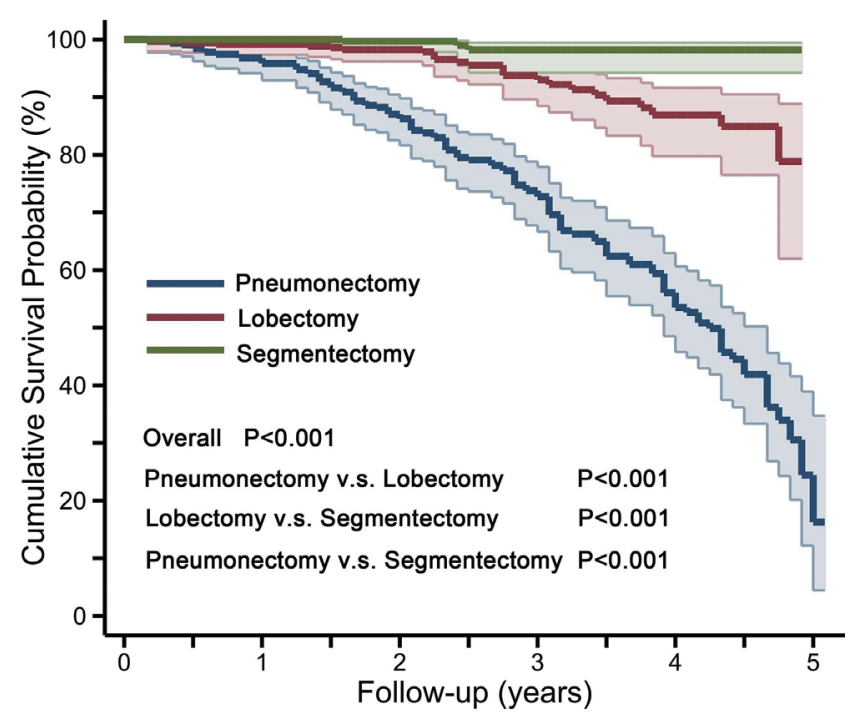

Figure 4 Kaplan-Meier survival curve showing post-discharge survival of patients who underwent pneumonectomy, lobectomy or segmentectomy, with $95 \%$ CI (shaded area). CI, confidence interval.

Also, the inconsistent findings of studies observing right atrial enlargement $(15,16)$ after pneumonectomy made it hard to ascribe POAF to right atrial stretch.

\section{Possible mechanism for higher POAF risk after pпeumonectomy}

Numerous studies have identified the importance of PV in the initiation and maintenance of $\mathrm{AF}(13,17)$, owing to its special anatomical and electrophysiological characteristics including (I) myocardial sleeve facilitating trigger activity; (II) the presence of pacemaker, Purkinje and transitional cells; and (III) unique distribution of ion channels (18). One distinguishing characteristic of pneumonectomy is that after removing one side of lung, all pulmonary circulation is forced to flow through the residual pulmonary vessels, increasing intra-PV pressure and PV wall tension, which probably facilitate POAF onset. Previous studies $(19,20)$ have documented that mechanical stretch on PV could induce AF by enhancing PV myocardium automaticity, slowing local conduction, and complicating myocardial activation, hence facilitating $\mathrm{AF}$ onset and maintenance. During pneumonectomy procedure, AF onset was observed after clamping the pulmonary artery, even before other stressors took effect, suggesting substantial AF-facilitating effect of hemodynamic change.
On the other hand, inflammation and ischemia adjacent to resection margin could remodel gap junction (21), increase local conduction heterogeneity and trigger AF (22). Therefore, from segmentectomy to pneumonectomy, POAF morbidity elevated remarkably as surgical scope expanded. A recent study also reported a lower POAF incidence in segmentectomy than in lobectomy (23).

Our EPS files showed that in AF patients with pneumonectomy history, PV stumps tend to be electrically active, possibly due to variable fibrosis in $\mathrm{PV}$ remnants and up-regulated autonomous nerve tone $(24,25)$. Moreover, remaining PVs could also serve as $\mathrm{AF}$ trigger, responsible for $\mathrm{AF}$ initiation and maintenance, further substantiating the importance of $\mathrm{PV}$ in $\mathrm{AF}$ after pneumonectomy. Because it would be inappropriate and risky to perform postoperative EPS, we could only indirectly assess AF mechanism for patients with lung resection history through EPS files.

\section{RFs for $P O A F$}

Previous studies have suggested that RFs for POAF following lung resection included advanced age, male sex, ischemic heart disease, valvular heart disease, heart failure, higher resected lung volume, intraoperative blood transfusion, and mediastinal lymph dissection $(4,5,26)$. Albeit speculation was made that pneumonectomy patients had higher POAF risk (5), further investigation was absent. To the best of our knowledge, this is the first large study that focused on POAF in pneumonectomy patients and observed significantly higher POAF risk within this subgroup. From an epidemiological perspective, human aging is associated with an increased possibility of developing AF (27), and advanced age is a widely validated RF for POAF in numerous studies (28). And LA enlargement tends to suggest underlying structural remodeling, which is deemed as the main contributor for initiation and persistence of AF (29). Therefore, it is reasonable that age $>60$ years and LAd $\geq 35 \mathrm{~mm}$ predicted POAF within this subgroup.

\section{Impact of POAF on clinical outcomes}

Although POAF predicts adverse clinical outcomes after cardiac surgery (30), it remains unclear if such correlation exists among pneumonectomy patients. In our study, POAF, along with infection and hemorrhage, was a predictor of death in pneumonectomy, raising mortality risk by 16 -fold relative to patients without POAF. And pneumonectomy patients with longer total $\mathrm{AF}$ duration and higher $\mathrm{AF}$ 
burden seemed to possess a potentially higher risk of perioperative death. The exact mechanism underlying this possible predictive effect is still unknown. POAF onset indicates that normal cardiac electrical activity is severely affected, which seemed irrelevant to other complications. Yet postoperative complications including POAF could be deemed a consequence of accumulated substrate exceeding patients' tolerance, including pre-existing substrate and surgery-related substrate such as inflammation, autonomous nervous system activation, stress and mechanical stimulus. And compared with lobectomy and segmentectomy, pneumonectomy group undertook greater surgical impact, with lower POAF threshold due to drastic pulmonary circulation change as a special surgery-induced substrate. Therefore, the presence of POAF may serve as an indicator for severe surgical-related impact which could induce fatal complications. Close observation and even precautionary measures should be applied to prevent adverse outcomes. Since pneumonectomy for pleural mesothelioma and benign diseases, such as tuberculosis destroyed lung, aspergilloma and bronchiectasis, share the same hemodynamic change as pneumonectomy for primary lung cancer, the implication of POAF could be the same.

However, POAF didn't predict adverse long-term outcomes after pneumonectomy. This is reasonable since POAF occurs with aggravation of surgery-induced stress, which gradually alleviates as patients' convalescence proceeds, therefore unable to affect long-term outcomes. In our study, senior age and advanced tumor stage predicted adverse longterm outcomes, which is consistent with a previous study (31).

\section{Limitations}

The present study has several limitations. Firstly, it is a retrospective, descriptive and non-randomized study. Secondly, patients undergoing lobectomy and segmentectomy were selected via stratified sampling, which might have introduced selection bias. Thirdly, the sample size of the present study is still limited in spite of considerable number of pneumonectomy patients. Fourthly, EPS files were not from the same cohort studied, in whom it was inappropriate to perform EPS right after lung resection; therefore, results from this assessment are only suggestive of a possible mechanism for POAF.

\section{Conclusions}

POAF in pneumonectomy occurred mostly as a solitary complication in-hospital, with higher incidence and risk than those for lobectomy and segmentectomy. Pneumonectomy patients aged $>60$ years or with $\operatorname{LAd} \geq 35 \mathrm{~mm}$ were at higher risk for POAF. Active PV potential originating from stump and non-stump PVs might potentially underlie POAF. POAF, along with infection and hemorrhage, was predictive of perioperative mortality in pneumonectomy.

\section{Acknowledgments}

Funding: This research was funded by a grant from the National Natural Science Foundation of China (No. 81970276); Cultivation Project for Municipal Hospital Clinical Research of Shenkang Hospital Development Center (No. SHDC21018X02).

\section{Footnote}

Reporting Checklist: The authors have completed the STROBE reporting checklist. Available at http://dx.doi. org/10.21037/jtd-20-1717

Data Sharing Statement: Available at http://dx.doi. org/10.21037/jtd-20-1717

Conflicts of Interest: All authors have completed the ICMJE uniform disclosure form (available at http://dx.doi. org/10.21037/jtd-20-1717). LZ reports grants from the National Natural Science Foundation of China, grants from Shenkang Hospital Development Center, during the conduct of the study. The other authors have no conflicts of interest to declare.

Ethical Statement: The authors are accountable for all aspects of the work in ensuring that questions related to the accuracy or integrity of any part of the work are appropriately investigated and resolved. The study protocol was approved by the Shanghai Chest Hospital Ethics Committee (ID: KS1846) and individual consent for this retrospective analysis was waived. The study was conducted in accordance with the Declaration of Helsinki (as revised in 2013).

Open Access Statement: This is an Open Access article distributed in accordance with the Creative Commons Attribution-NonCommercial-NoDerivs 4.0 International License (CC BY-NC-ND 4.0), which permits the noncommercial replication and distribution of the article with 
the strict proviso that no changes or edits are made and the original work is properly cited (including links to both the formal publication through the relevant DOI and the license). See: https://creativecommons.org/licenses/by-nc-nd/4.0/.

\section{References}

1. Bray F, Ferlay J, Soerjomataram I, et al. Global cancer statistics 2018: GLOBOCAN estimates of incidence and mortality worldwide for 36 cancers in 185 countries. CA Cancer J Clin 2018;68:394-424.

2. Howington JA, Blum MG, Chang AC, et al. Treatment of stage I and II non-small cell lung cancer: diagnosis and management of lung cancer, 3rd ed: American College of Chest Physicians evidence-based clinical practice guidelines. Chest 2013;143:e278S-313S.

3. Roselli EE, Murthy SC, Rice TW, et al. Atrial fibrillation complicating lung cancer resection. J Thorac Cardiovasc Surg 2005;130:438-44.

4. Muranishi $Y$, Sonobe M, Menju T, et al. Atrial fibrillation after lung cancer surgery: incidence, severity, and risk factors. Surg Today 2017;47:252-8.

5. Onaitis $\mathrm{M}, \mathrm{D}$ 'Amico $\mathrm{T}$, Zhao $\mathrm{Y}$, et al. Risk factors for atrial fibrillation after lung cancer surgery: analysis of the Society of Thoracic Surgeons general thoracic surgery database. Ann Thorac Surg 2010;90:368-74.

6. Ishibashi H, Wakejima R, Asakawa A, et al. Postoperative atrial fibrillation in lung cancer lobectomy-analysis of risk factors and prognosis. World J Surg 2020;44:3952-9.

7. Amar D, Zhang H, Tan KS, et al. A brain natriuretic peptide-based prediction model for atrial fibrillation after thoracic surgery: development and internal validation. J Thorac Cardiovasc Surg 2019;157:2493-9.e1.

8. Lee SH, Ahn HJ, Yeon SM, et al. Potentially modifiable risk factors for atrial fibrillation following lung resection surgery: a retrospective cohort study. Anaesthesia 2016;71:1424-30.

9. Smith H, Li H, Brandts-Longtin O, et al. External validity of a model to predict postoperative atrial fibrillation after thoracic surgery. Eur J Cardiothorac Surg 2020;57:874-80.

10. Dobrev D, Aguilar M, Heijman J, et al. Postoperative atrial fibrillation: mechanisms, manifestations and management. Nat Rev Cardiol 2019;16:417-36.

11. Iwata T, Nagato K, Nakajima T, et al. Risk factors predictive of atrial fibrillation after lung cancer surgery. Surg Today 2016;46:877-86.

12. Chen LY, Chung MK, Allen LA, et al. Atrial fibrillation burden: moving beyond atrial fibrillation as a binary entity: a scientific statement from the American Heart Association. Circulation 2018;137:e623-44.

13. Haïssaguerre M, Jaïs P, Shah DC, et al. Spontaneous initiation of atrial fibrillation by ectopic beats originating in the pulmonary veins. N Engl J Med 1998;339:659-66.

14. Heijman J, Guichard JB, Dobrev D, et al. Translational challenges in atrial fibrillation. Circ Res 2018;122:752-73.

15. Neragi-Miandoab S, Weiner S, Sugarbaker DJ. Incidence of atrial fibrillation after extrapleural pneumonectomy vs. pleurectomy in patients with malignant pleural mesothelioma. Interact Cardiovasc Thorac Surg 2008;7:1039-42.

16. Kowalewski J, Brocki M, Dryjanski T, et al. Right ventricular morphology and function after pulmonary resection. Eur J Cardiothorac Surg 1999;15:444-8.

17. Oral H, Pappone C, Chugh A, et al. Circumferential pulmonary-vein ablation for chronic atrial fibrillation. $\mathrm{N}$ Engl J Med 2006;354:934-41.

18. Khan R. Identifying and understanding the role of pulmonary vein activity in atrial fibrillation. Cardiovasc Res 2004;64:387-94.

19. Walters TE, Lee G, Spence S, et al. Acute atrial stretch results in conduction slowing and complex signals at the pulmonary vein to left atrial junction: insights into the mechanism of pulmonary vein arrhythmogenesis. Circ Arrhythm Electrophysiol 2014;7:1189-97.

20. Hamaguchi S, Hikita K, Tanaka Y, et al. Enhancement of automaticity by mechanical stretch of the isolated guinea pig pulmonary vein myocardium. Biol Pharm Bull 2016;39:1216-9.

21. Kondo H, Takahashi N, Gotoh K, et al. Splenectomy exacerbates atrial inflammatory fibrosis and vulnerability to atrial fibrillation induced by pressure overload in rats: Possible role of spleen-derived interleukin-10. Heart Rhythm 2016;13:241-50.

22. Rena O, Papalia E, Oliaro A, et al. Supraventricular arrhythmias after resection surgery of the lung. Eur J Cardiothorac Surg 2001;20:688-93.

23. Ueda T, Suzuki K, Matsunaga T, et al. Postoperative atrial fibrillation is less frequent in pulmonary segmentectomy compared with lobectomy. Gen Thorac Cardiovasc Surg 2018;66:95-100.

24. Kanmanthareddy A, Vallakati A, Reddy Yeruva M, et al. Pulmonary vein isolation for atrial fibrillation in the postpneumonectomy population: a feasibility, safety, and outcomes study. J Cardiovasc Electrophysiol 2015;26:385-9.

25. Sasaki N, Okumura Y, Watanabe I, et al. Pulmonary vein remnant as a trigger site for atrial fibrillation. Circ J 
2013;77:494-6.

26. Echahidi N, Pibarot P, O'Hara G, et al. Mechanisms, prevention, and treatment of atrial fibrillation after cardiac surgery. J Am Coll Cardiol 2008;51:793-801.

27. Miyasaka Y, Barnes ME, Gersh BJ, et al. Secular trends in incidence of atrial fibrillation in Olmsted County, Minnesota, 1980 to 2000, and implications on the projections for future prevalence. Circulation 2006;114:119-25.

28. Amar D. Postoperative atrial fibrillation: is there a need for prevention? J Thorac Cardiovasc Surg 2016;151:913-5.

29. Sanders P, Morton JB, Davidson NC, et al. Electrical

Cite this article as: Wang $\mathrm{H}$, Wang Z, Zhou M, Chen J, Yao F, Zhao L, He B. Postoperative atrial fibrillation in pneumonectomy for primary lung cancer. $J$ Thorac Dis 2021;13(2):789-802. doi: 10.21037/jtd-20-1717 remodeling of the atria in congestive heart failure: electrophysiological and electroanatomic mapping in humans. Circulation 2003;108:1461-8.

30. Phan K, Ha HS, Phan S, et al. New-onset atrial fibrillation following coronary bypass surgery predicts long-term mortality: a systematic review and meta-analysis. Eur J Cardiothorac Surg 2015;48:817-24.

31. Kim DJ, Lee JG, Lee CY, et al. Long-term survival following pneumonectomy for non-small cell lung cancer: clinical implications for follow-up care. Chest 2007;132:178-84. 


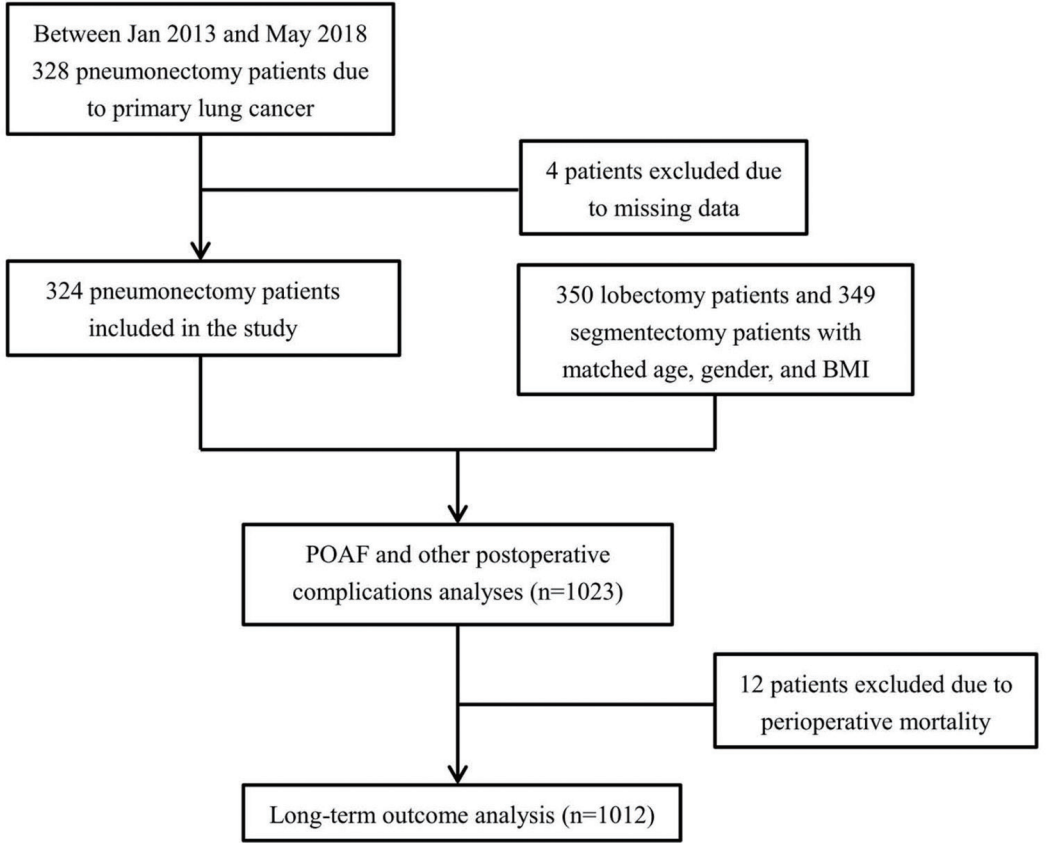

Figure S1 Flow diagram of patient selection process. BMI, body mass index; POAF, postoperative atrial fibrillation. 